Journal of Nuclear Medicine, published on February 26, 2021 as doi:10.2967/jnumed.120.256396

\title{
Direct Attenuation Correction Using Deep Learning for Cardiac SPECT: A Feasibility Study
}

Jaewon Yang ${ }^{1}$, Luyao Shi², Rui Wang², Edward J. Miller², Albert J. Sinusas², Chi Liu², Grant T. Gullberg ${ }^{1}$, and Youngho Seo ${ }^{1}$

${ }^{1}$ Physics Research Laboratory, Department of Radiology and Biomedical Imaging, University of California San Francisco, CA, USA

${ }^{2}$ Radiology and Biomedical Imaging, Yale University, New Haven, CT, USA

Corresponding author: Jaewon Yang, PhD

UCSF Physics Research Laboratory

Email: jaewon.yang@ucsf.edu

Phone: 650-644-6045

Running Title: Direct AC using DL for SPECT MPI

Keywords: cardiac SPECT, MPI, attenuation correction, deep learning 


\section{ABSTRACT}

Purpose: Dedicated cardiac SPECT scanners with cadmium-zinc-telluride (CZT) cameras have shown capabilities of shortened scan times or reduced radiation doses as well as improved image quality. Since most of the dedicated scanners do not have an integrated $\mathrm{CT}$, image quantification with attenuation correction $(A C)$ is challenging and artifacts are routinely encountered in daily clinical practice. In this work, we demonstrate a direct AC technique using deep learning (DL) for myocardial perfusion imaging (MPI).

Methods: In an IRB-approved retrospective study, 100 cardiac SPECT/CT datasets with ${ }^{99 m}$ Tctetrofosmin using a GE Discovery NM/CT 570c scanner were collected at the Yale New Haven Hospital. A U-Net-based network was used for generating attenuation-corrected SPECT (SPECTDL) directly from non-corrected SPECT (SPECT $T_{N C}$ ) without undergoing an additional image reconstruction step. The accuracy of SPECTDL was evaluated by voxel-wise and segment-wise analyses against the reference CT-based AC (SPECTCTAC) using American Heart Association 17 segments in the myocardium. Polar maps of representative (best/median/worst) cases were visually compared for illustrating potential benefits and pitfalls of the DL approach.

Results: The voxel-wise correlations with SPECTCTAC were $92.2 \% \pm 3.7$ (slope $=0.87 ; R^{2}=$ 0.81 ) and $97.7 \% \pm 1.8$ (slope $=0.94 ; R^{2}=0.91$ ) for SPECT SC and SPECT $D L$, respectively. The segmental errors of SPECT $N$ scattered from $-35 \%$ up to $21 \%(p<0.001)$; while, the errors of SPECTDL stayed mostly within $\pm 10 \%(p<0.001)$. The average segmental errors (mean $\pm S D)$ were $-6.11 \pm 8.06 \%$ and $0.49 \pm 4.35 \%$ for SPECT $N C$ and SPECTDL, respectively. The average absolute segmental errors were $7.96 \pm 6.23 \%$ and $3.31 \pm 2.87 \%$ for SPECT SC and SPECTDL, respectively. Review of polar maps revealed successful demonstration of reduced attenuation artifacts; however, the performance of SPECTDL was not consistent for all subjects likely due to different amount of attenuation and uptake patterns.

Conclusion: We demonstrated the feasibility of direct AC using DL for SPECT MPI. Overall, our $\mathrm{DL}$ approach reduced attenuation artifacts substantially compared to SPECT $\mathrm{NC}_{\mathrm{NC}}$, justifying further studies to establish safety and consistency for clinical applications in stand-alone SPECT systems suffered from attenuation artifacts. 


\section{INTRODUCTION}

SPECT myocardial perfusion imaging (MPI) is the most widely applied noninvasive method for the detection and risk stratification of coronary artery disease (1). It is less costly and more widely available than PET MPI. However, since conventional SPECT systems are not optimized for cardiac imaging, dedicated cardiac SPECT scanners with cadmium-zinc-telluride (CZT) cameras have been developed constraining the entire detector area to imaging just the heart (2). The CZT cameras can achieve 5-10 times higher count sensitivity and 3-6 times faster scanning times than conventional sodium iodide SPECT cameras (3), which can result in higher image quality with reduced radiation dose as well as promote easier scheduling and higher patient satisfaction (2).

However, artifacts of dedicated cardiac SPECT cameras are commonly observed in daily clinical practice as for conventional SPECT cameras (4). Soft tissue attenuation is the most common cause for the artifacts usually due to attenuation caused by the diaphragm, breast tissue or obesity (5). Therefore, it is encouraged to incorporate of attenuation correction (AC) into routine clinical practice in order to reduce the artifacts and thus improve diagnostic accuracy (6). Nevertheless, AC is not performed as a routine clinical practice for most of the dedicated cardiac SPECT systems not integrated with a CT (7).

AC techniques for SPECT are categorized into three classes according to how to generate attenuation maps. The first approach is to perform transmission CT scans for generating AC maps (called CTAC), which is not available in stand-alone SPECT systems occupying approximately $80 \%$ of the market share (8) but only in integrated SPECT/CT systems. The second approach is to use line sources (most commonly Gd-153), which may cause lowcount AC maps and truncation artifacts (due to obesity) or cross-talk with emission data (9). The third approach is to estimate AC maps from emission data, which is also classified into segmentation-based (10) and model-based methods (11) but not practical due to timeconsuming process or computation.

Recently, it was demonstrated that attenuation maps could be directly estimated from the emission data using a deep learning (DL) model (12). In general, DL applications for AC have been actively investigated in PET/MRI to convert MR images directly to pseudo CT $(13,14)$. Furthermore, a direct DL approach was proposed to convert non-corrected PET to corrected PET in image space without any additional reconstruction steps $(15,16)$, which has not been applied to SPECT yet. Also, an indirect approach was demonstrated for stand-alone conventional SPECT scanners (12), requiring the reconstruction of full field-of-view (FOV) 
emission images for generating full FOV attenuation maps. For this reason, such indirect approach of generating attenuation maps as an intermediate step cannot be directly applied to dedicated cardiac SPECT scanners such as GE Discovery NM 530c whose geometry is unique with a small FOV of about $19 \mathrm{~cm}$ in diameter (17). Therefore, a direct conversion approach such as our work is needed specifically for the scanner with a small FOV. It is worth noting that our $\mathrm{DL}$ approach cannot be directly compared with any indirect $\mathrm{DL}$ approach such as (12).

Therefore, we demonstrate a direct $\mathrm{DL}$ technique for CT-less $\mathrm{AC}$ in dedicated cardiac SPECT systems. Considering AC as a computer vision task, we hypothesize that DL can recognize clinically relevant uptake patterns in both non-corrected and corrected images for removing attenuation-involved artifacts in SPECT MPI.

\section{MATERIALS AND METHODS}

\section{SPECT Datasets}

The institutional review board (IRB) approved this retrospective study and the requirement to obtain informed consent was waived. 100 datasets ( 58 male and 42 female) were collected from cardiac stress-only ${ }^{99 m}$ Tc-tetrofosmin SPECT using a GE Discovery NM/CT 570c (GE D570c) scanner at Yale New Haven Hospital. The characteristics of subjects are not available due to the deidentification performed for the data transfer to UCSF. CT (120 kVp, $50 \mathrm{~mA}$ and rotation time of $0.4 \mathrm{~s}$ ) images were aligned with non-corrected SPECT (SPECTNC) images in the myocardium region through the Attenuation Correction Quality Control package routinely used in clinical practice. Using a one-step-late algorithm with Green prior, image reconstructions (70x70x50 matrix size with $4 \mathrm{~mm}$ voxel size) were performed with 30 iterations and post-filtering ( $10^{\text {th }}$ order Butterworth filter, $0.4 \mathrm{~cm}^{-1}$ cutoff) for SPECT $\mathrm{NC}_{\mathrm{NC}}$ and 60 iterations and post-filtering ( $7^{\text {th }}$ order Butterworth filter, $0.37 \mathrm{~cm}^{-1}$ cutoff) for SPECT with CTAC (SPECTCTAC). All the reconstruction and filter parameters are clinically used but no established scatter correction is being routinely applied for GE D570c scanners with CZT detectors at Yale New Haven Hospital.

\section{Deep Convolutional Neural Network (DCNN)}

The proposed DL model aims to transform SPECT NC $_{\text {Co }}$ SPECTCTAC directly in image space (SPECT $\mathrm{DL}$ ), without generating $\mu$-maps for conventional image reconstruction combined with $\mathrm{AC}$ (Figure 1). The proposed DCNN is an extended three dimensional version of our previous work (15). Different from conventional scanners, dedicated cardiac SPECT scanners allow a limited FOV to include at least the whole heart, which enables ideal three dimensional model training 
without separating the left ventricle to smaller patches. The original patch size of $70 \times 70 \times 50$ was reduced to $64 \times 64 \times 32$ by removing the edges of each patch for enabling down-sampling $(64 \rightarrow$ $32 \rightarrow \cdots \rightarrow 4$ ) in each dimension. Our DCNN consists of five encoder-decoder stages symmetrically concatenated with skip connections. In each stage, convolution with $3 \times 3 \times 3$ kernels, batch normalization, and rectified linear unit is sequentially performed twice. Between stages, the downsampling and upsampling are done by $2 \times 2 \times 2$ max pooling and bilinear interpolation, respectively. The original design adapted from our previous work (15) was modified to a 3D network architecture for this study.

\section{DCNN Training and Testing}

The DCNN was trained and tested with paired input (SPECT ${ }_{N C}$ ) and output (SPECTCTAC) patches using 10-folded cross validation without using external validation: i.e., the model was trained with 9 groups (10 in each group) and tested with the other one, which was repeated 10 times. The raw values (absolute counts) of each patch were normalized by its maximum to reduce the dynamic range of input/output values. In the patches, low counts out of the myocardium were considered background noise and removed by binary masking (15). Before being fed into the model, 90 input patches were randomly shuffled, rotated ( $\leq 15$ degree in transaxial view), and translated horizontally ( $\leq 10$ pixels), vertically ( $\leq 10$ pixels) and axially ( $\leq 3$ pixels) for data augmentation. A learning rate initialized by 0.001 was reduced to $3 / 4^{\text {th }}$ of its current value in the course of training if the loss did not decrease in 25 epochs. Weights for convolution were initialized with the He-initializer (18) and all biases were initialized with zero. Mean squared error (or L2 loss) and RMSprop optimizer (19) were used for optimizing weights and biases. The loss was converged in 1500 epochs and the training was stopped the point, which was empirically determined through loss curves and consistent across all the folds. The hyper parameters empirically chosen in our previous work (15) were adapted for this study through hyperparameter tuning.

The proposed model was implemented using Tensorflow (version 1.12.0) and Keras libraries (version 2.2.4). Model training and testing were performed on a Ubuntu server with a single Tesla V100 (NVIDIA) graphics processing unit. The training took approximately 100 minutes to reach stability. After training the model, it takes only $0.5 \mathrm{~s}$ on average to generate SPECTDL volumetric images $(64 \times 64 \times 32)$. 


\section{Quantitative Analysis}

Overall quantitative accuracy of SPECTDL was evaluated, compared to reference SPECTCTAC. For voxel-wise analysis, the normalized root mean square error, peak signal to noise ratio and structural similarity index were quantified. For segment-wise analysis, polar maps were generated and compared using a 17-segment model for the left ventricle, which was adopted from (20). A measured count of each segment was normalized by the maximum count of 17 segments. The relative percentage count of each segment was computed and an error (i.e., SPECTDL - SPECTCTAC) was assessed. Additionally, the effects of sex (58 males versus 42 females) and hepatic uptake (44 subjects with high liver uptake versus 56 subjects with low liver uptake) were investigated through the segment-wise analysis separately for each cohort. Although the information of patient weight and height was not available for computing body mass index, the volume of each patient was estimated from CT-derived attenuation maps for investigating any correlation between chest volumes and average segmental errors across subjects.

\section{Illustration of Representative Cases}

Three categories of subjects were selected to illustrate the overall qualitative assessment of SPECTDL. First, three subjects with the smallest mean segmental error were selected to show examples of SPECTDL that achieved quantitatively the most accurate correction. Second, three subjects with the $25^{\text {th }}, 50^{\text {th }}$ and $75^{\text {th }}$ percentiles of absolute mean segmental errors were chosen to depict examples that achieved quantitatively median accuracy. Finally, two subjects with the highest and lowest mean segmental errors were selected to display examples that our DL model was not able to correct accurately.

\section{Statistics}

A joint histogram was employed to show the distribution of voxel-wise correlation of SPECTDL with reference SPECTCTAC. Also, the error distributions of all segments of all 100 subjects $(17 \times$ $100=1700$ segments in total) for SPECTDL were displayed in Bland-Altman and box plots. A paired $t$ test was performed for comparing the segmental uptake values of SPECTDL with those of the reference. A $P$ value of less than 0.05 was considered to indicate statistical significance.

Polar maps were generated using the PMOD Cardiac PET Modeling tool (PCARDP, version 3.8). All the processing and analyses above were performed in MATLAB (R2015b, MathWorks). 


\section{RESULTS}

For voxel-wise analysis, the normalized root mean square errors were $0.232 \pm 0.077$ and 0.148 \pm 0.095 ; the peak signal to noise ratios were $31.3 \pm 2.8$ and $36.2 \pm 4.1$; the structural similarity indices were $0.984 \pm 0.008$ and $0.993 \pm 0.006$ for SPECTNC and SPECTDL, respectively. Consistently, the voxel-wise correlations were $92.2 \% \pm 3.7$ (slope $=0.87 ; R^{2}=0.81$ ) and $97.7 \%$ \pm 1.8 (slope $=0.94 ; R^{2}=0.91$ ) for SPECT NC and SPECTDL, respectively (Figure 2 ).

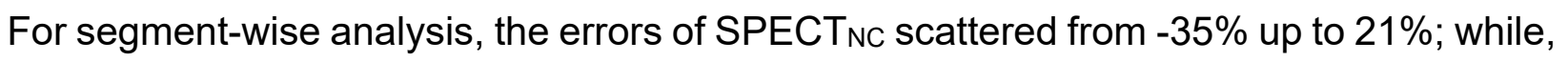
those of SPECTDL stayed mostly within $\pm 10 \%$ (Figure 3 ). Consequently, the average errors (mean \pm SD) were $-6.11 \pm 8.06 \%$ and $0.49 \pm 4.35 \%$; and the average absolute errors were 7.96 $\pm 6.23 \%$ and $3.31 \pm 2.87 \%$ for SPECT NC and SPECT DL , respectively, which were consistent with the sex-dependent and hepatic uptake-dependent results where the average errors of SPECT $T_{N C}$ were substantially reduced through $\mathrm{DL}$ (Table 1 ). All results were statistically significant except for male $(p=0.10)$ and subjects with HLU $(p=0.25)$.

Figure 4 shows that $\mathrm{DL}$ reduced the error distributions of all segments close to zero mean within $\pm 10 \%$ variation across all subjects, which was consistent with the corresponding error distributions for male, female and subjects with HLU and LLU (Supplementary Figure 1). Specifically, DL reduced the average absolute error of SPECT NC $_{\text {by }}>70 \%$ for the segments of $4,9,10,15$ (segment \#); > $60 \%$ for the segments of $3,5,11$; > $50 \%$ for the segments of 8,$14 ;>$ $40 \%$ for the segments of 16,17 ; and $>17 \%$ for the rest. The box plot of each subject across all 17 segments was found in Supplementary Figure 2. In the analysis of correlation between patients' chest volumes and average segmental errors, no correlation was found for both $\operatorname{SPECT}_{N C}(\rho=0.016)$ and SPECTDL $(\rho=0.021)$, though a moderate non-linear (affine) correlation ( $\rho=0.6264$ ) was found between SPECT NC and SPECTDL (Supplementary Figure 3).

Three categories of subjects were described in Table 2 for illustrating the overall performance of our DL approach. Figure 5 illustrates accurately corrected attenuation artifacts in the right coronary artery (RCA) territory of SPECTDL. In Figure 6, artifacts were accurately corrected in first two cases $\left(25^{\text {th }}\right.$ and $50^{\text {th }}$ percentiles) in the RCA of SPECTDL, though a remarkable visual difference between SPECTNC and SPECTCTAC was not observed for the last case (75th percentile). Figure 7 illustrates two cases with substantially overestimated SPECTDL in the RCA and left circumflex artery (LCX) and substantially underestimated SPECTDL in the RCA and left anterior descending artery (LAD). 


\section{DISCUSSION}

We demonstrated the our DL model substantially reduced the attenuation artifacts of SPECTNC, illustrating its potential benefits and pitfalls. To our knowledge, this is the first work to demonstrate a CT-less direct AC in image space, as an accelerated one-step process for for SPECT MPI in a dedicated SPECT system.

There are several benefits of our DL approach technically and clinically for SPECT MPI. From technical perspective, the biggest potential advantage is to remove the need of attenuation map generation that is an essential step of conventional image reconstruction. Most dedicated cardiac SPECT systems (7) and approximately $80 \%$ of SPECT systems in the market are standalone (i.e., not combined with a CT) (8), which acknowledges the potential value of our DL approach. Also, our direct DL approach is much simpler and faster $(<1 \mathrm{~s})$ than conventional approaches that require an additional reconstruction step with time-consuming computation. From clinical perspective, when approaches such as conventional AC methods or alternative prone imaging are not possible (5), our DL model could potentially incorporate AC into routine clinical practice (6) and efficiently improve the accuracy of diagnosis in existing stand-alone SPECT systems, since attenuation artifacts due to soft-tissue are more likely to be misinterpreted as fixed defects in normal patients with normal heart function (21). Also, our model trained with datasets acquired in a conventional SPECT/CT scanner could be applied to different datasets acquired in a stand-alone scanner if images are reconstructed with similar parameters and their pixel sizes are consistent across two different scanners. For example, it would be feasible to apply our DL model trained with datasets from a GE D570c (combined SPECT/CT) to new datasets acquired in a GE D530c (stand-alone) since the geometries and hardware components of the two scanners are very close and thus their non-corrected image qualities are similar. Nevertheless, it is expected that an indirect $D L$ approach such as generating pseudo CT (12) would perform more consistently than our direct DL approach, since the indirect approach keeps every aspect of the conventional image reconstruction; however, a direct approach like ours needs to learn attenuation physics and statistics by a DCNN, which requires large normative training data. Another important clinical merit of our CT-less DL approach is the reduction of radiation dose from $\mathrm{CT}$ that may lead to a substantial increase in lifetime cancer risk (9), specifically for pediatric patients who are much sensitive to radiation $(10,11)$.

The general rules of attenuation in SPECT MPI were derived from the literatures as follows (5). First, in male more often than in female, the left hemidiaphragm results in potential perfusion artifacts in the RCA, that is more accentuated with abdominal protuberance, elevated 
left hemidiaphragm, or obesity; Second, in female more often than in male, breast attenuation causes potential perfusion artifacts in the LAD or LCx; Third, the high-uptake liver may create artifacts in the inferior wall. Our result was consistent with the general rules in many aspects. Overall, Figure 4 confirmed the most important first rule that large errors were mostly found in the RCA of SPECTNC but corrected in SPECTDL: Our DL approach substantially reduced artifacts across all RCA, LAC and LCx territories. The errors of SPECTNC in the LAD and LCx were relatively much smaller than those in the RCA for both male and female (Supplementary Figure $1 \mathrm{~A}$ ), implying that breast attenuation ( $2^{\text {nd }}$ rule) was not dominant in our datasets, and the slightly higher error of male for SPECT $N$ N was observed, which might be inferred from the general characteristic of men taller and heavier than women (22) but were not able to be thoroughly explained due to the missing information of subject height and weight. The larger average error of subjects with HLU for SPECTNC (Supplementary Figure 1B) was consistent with the $3^{\text {rd }}$ rule.

There is always risk that any new DL models may miss important patterns or generate pseudo patterns when exposed to new test data with different characteristics (23). Thus, representative cases were selected according to subject-specific quantitative results, which was appropriate and desirable for illustrating specific (best/median/worst) cases. The visual performance of our DL approach was not consistent across all subjects likely due to different amount of soft tissue and uptake patterns, that was inferred from the literatures demonstrating substantially degraded image quality of SPECT ${ }_{N C}$ for obese patients (5); however, at least in our dataset, there was no correlation between patients' chest volumes and average errors for both SPECT $_{N C}$ and SPECT $D L$ (Supplementary Figure 3). From the perspective of model training, since our DL model was optimized only in our limited dataset, the current model might not be able to achieve high accuracy for such cases (Figure 7) whose attenuation artifacts might be unique and very different from the artifacts of other subjects. Therefore, there is still room to improve the accuracy of our model towards clinical translation.

Despite the promising results, our study has several limitations. First, 100 subjects may not be enough to represent all possible patterns involved with attenuation artifacts. For example, the female population might not contain significant breast attenuation as discussed above, which implies the need of constructing a large dataset with more diverse cases for future studies. Second, the clinical information (weight, height, clinical interpretation, etc.) was missing due to the deidentification, and a thorough investigation of their effects on the current results is beyond the scope of this study. Third, any observer study or clinical interpretation (e.g., scoring SPECTDL verse SPECT CTAC) $_{\text {) }}$ was not performed, though summed perfusion scores derived from a normal 
dataset would improve the quality of this study; however, the uptake distribution of SPECTCTAC (i.e., reference standard) was not uniform as shown in the x-axis of the Bland-Altman plot (Figure 3 ) and thus a normal dataset was not able to be derived for the scoring. Fourth, SPECTCTAC might include unexpected artifacts because of uncorrected scatter produced by subdiaphragmatic high uptake organs (24). Unfortunately, conventional scatter correction approaches are not applicable to GE D570c scanners due to the unique energy spectrum of pixelated CZT detectors with a tailing effect caused by incomplete charger and inter-detector scatters, thus clinical adoption of CZT-specific scatter correction methods are needed in the future $(25,26)$. Finally, the right ventricle was unseen in SPECTDL since low-count voxels out of the myocardium were considered background noise and removed by binary masking; however, an advanced network architecture might enable to skip the binary masking.

Therefore, for future work, it would be important to construct a large normative database with more diverse and outlier cases for investigating the effects of patient characteristics on model training and evaluation, deleting suspicious data with unexpected artifacts based on physicians' interpretation. Also, it would be of great value to develop a validation program for evaluating large test datasets efficiently using automatic myocardium segmentation (27) and image quality assessment (28), which could accelerate quantitative and qualitative analyses. These future efforts would establish safety and consistency required for reliable interpretation towards clinical translation.

\section{CONCLUSION}

We demonstrated the feasibility of direct AC using DL for SPECT MPI, illustrating potential benefits and pitfalls. Overall, the DL model reduced attenuation artifacts substantially compared to SPECT ${ }_{N C}$, justifying further studies to establish safety and consistency for clinical applications in stand-alone SPECT systems suffered from attenuation artifacts. 


\section{ACKNOWLEDGMENTS}

The study was supported by the National Institutes of Health under Grants R01HL135490 and R01EB026331, R01HL123949, and American Heart Association award 18PRE33990138.

\section{No potential conflicts of interest relevant to this article exist.}

\section{KEY POINTS}

QUESTION: Is it feasible to employ deep learning (DL) for CT-less attenuation correction (AC) in image space for myocardial perfusion imaging (MPI) in a dedicated cardiac SPECT system? PERTINENT FINDINGS: In a cohort study evaluating DL-based AC in 100 patients undergoing SPECT MPI with and without AC, our DL approach achieved quantitative and qualitative accuracy comparable to the conventional CT-based AC, reducing attenuation artifacts observed in non-corrected SPECT.

\section{IMPLICATIONS FOR PATIENT CARE:}

This DL technique demonstrated its potential to efficiently reduce attenuation artifacts observed in stand-alone SPECT systems for improving sensitivity and specificity in clinical interpretation. 


\section{REFERENCES}

1. Hage FG, AlJaroudi WA. Review of cardiovascular imaging in the Journal of Nuclear Cardiology in 2017. Part 2 of 2: Myocardial perfusion imaging. J Nucl Cardiol. 2018;25:1390-1399.

2. Garcia EV, Faber TL, Esteves FP. Cardiac dedicated ultrafast SPECT cameras: new designs and clinical implications. J Nucl Med. 2011;52:210-217.

3. Esteves FP, Raggi P, Folks RD, et al. Novel solid-state-detector dedicated cardiac camera for fast myocardial perfusion imaging: multicenter comparison with standard dual detector cameras. $J$ Nucl Cardiol. 2009;16:927-934.

4. Kao YH, Better N. D-SPECT: New technology, old tricks. J Nucl Cardiol. 2016;23:311-312.

5. Singh B, Bateman TM, Case JA, Heller G. Attenuation artifact, attenuation correction, and the future of myocardial perfusion SPECT. J Nucl Cardiol. 2007;14:153-164.

6. Heller GV, Links J, Bateman TM, et al. American Society of Nuclear Cardiology and Society of Nuclear Medicine joint position statement: attenuation correction of myocardial perfusion SPECT scintigraphy. J Nucl Cardiol. 2004;11:229-230.

7. Allie R, Hutton BF, Prvulovich E, Bomanji J, Michopoulou S, Ben-Haim S. Pitfalls and artifacts using the D-SPECT dedicated cardiac camera. J Nucl Cardiol. 2016;23:301-310.

8. 2017. TGSM-. https://wwwtechnaviocom/report/global-medical-imaging-global-spectmarket-2017-2021. Accessed 2 Jun, 2020.

9. Pazhenkottil AP, Kaufmann PA, Gaemperli O. Attenuation correction in stress-only myocardial perfusion imaging. J Nucl Cardiol. 2017;24:402-404.

10. Nunez M, Prakash V, Vila R, Mut F, Alonso O, Hutton BF. Attenuation correction for lung SPECT: evidence of need and validation of an attenuation map derived from the emission data. Eur J Nucl Med Mol Imaging. 2009;36:1076-1089.

11. Cade SC, Arridge S, Evans MJ, Hutton BF. Use of measured scatter data for the attenuation correction of single photon emission tomography without transmission scanning. Med Phys. 2013;40:082506.

12. Shi L, Onofrey JA, Liu H, Liu YH, Liu C. Deep learning-based attenuation map generation for myocardial perfusion SPECT. Eur J Nucl Med Mol Imaging. 2020;47:2383-2395.

13. Leynes AP, Yang J, Wiesinger F, et al. Zero-Echo-Time and Dixon Deep Pseudo-CT (ZeDD CT): Direct Generation of Pseudo-CT Images for Pelvic PET/MRI Attenuation Correction Using Deep Convolutional Neural Networks with Multiparametric MRI. J Nucl Med. 2018;59:852-858.

14. Gong K, Yang J, Kim K, El Fakhri G, Seo Y, Li Q. Attenuation correction for brain PET imaging using deep neural network based on Dixon and ZTE MR images. Phys Med Biol. 2018;63:125011. 
15. Yang J, Park D, Gullberg GT, Seo Y. Joint correction of attenuation and scatter in image space using deep convolutional neural networks for dedicated brain (18)F-FDG PET. Phys Med Biol. 2019;64:075019.

16. Shiri I, Arabi H, Geramifar P, et al. Deep-JASC: joint attenuation and scatter correction in whole-body (18)F-FDG PET using a deep residual network. Eur J Nucl Med Mol Imaging. 2020;47:2533-2548.

17. Wu J, Liu C. Recent advances in cardiac SPECT instrumentation and imaging methods. Phys Med Biol. 2019;64:06TR01.

18. He K, Zhang X, Ren S, Sun J. Delving deep into rectifiers: surpassing human-level performance on ImageNet classification. arXiv:150201852. 2015.

19. Hinton G, Srivastava N, Swersky K. Neural Networks for Machine Learning Lecture 6e rmsprop: Divide the gradient by a running average of its recent magnitude. Accessed 2 Jun, 2020.

20. Herzog BA, Buechel RR, Husmann L, et al. Validation of CT attenuation correction for highspeed myocardial perfusion imaging using a novel cadmium-zinc-telluride detector technique. $J$ Nucl Med. 2010;51:1539-1544.

21. DePuey EG, Rozanski A. Using gated technetium-99m-sestamibi SPECT to characterize fixed myocardial defects as infarct or artifact. J Nucl Med. 1995;36:952-955.

22. Ogden CL, Fryar CD, Carroll MD, Flegal KM. Mean body weight, height, and body mass index, United States 1960-2002. Adv Data. 2004:1-17.

23. Zaharchuk G. Next generation research applications for hybrid PET/MR and PET/CT imaging using deep learning. Eur J Nucl Med Mol Imaging. 2019;46:2700-2707.

24. Dvorak RA, Brown RK, Corbett JR. Interpretation of SPECT/CT myocardial perfusion images: common artifacts and quality control techniques. Radiographics. 2011;31:2041-2057.

25. Pourmoghaddas A, Vanderwerf K, Ruddy TD, Glenn Wells R. Scatter correction improves concordance in SPECT MPI with a dedicated cardiac SPECT solid-state camera. J Nucl Cardiol. 2015;22:334-343.

26. Fan $\mathrm{P}$, Hutton $\mathrm{BF}$, Holstensson $\mathrm{M}$, et al. Scatter and crosstalk corrections for $(99 \mathrm{~m}) \mathrm{Tc} /(123) \mathrm{I}$ dual-radionuclide imaging using a CZT SPECT system with pinhole collimators. Med Phys. 2015;42:6895-6911.

27. Wang $\mathrm{T}$, Lei $\mathrm{Y}$, Tang $\mathrm{H}$, et al. A learning-based automatic segmentation and quantification method on left ventricle in gated myocardial perfusion SPECT imaging: A feasibility study. J Nucl Cardiol. 2020;27:976-987. 
28. Renieblas GP, Nogues AT, Gonzalez AM, Gomez-Leon N, Del Castillo EG. Structural similarity index family for image quality assessment in radiological images. J Med Imaging (Bellingham). 2017;4:035501. 


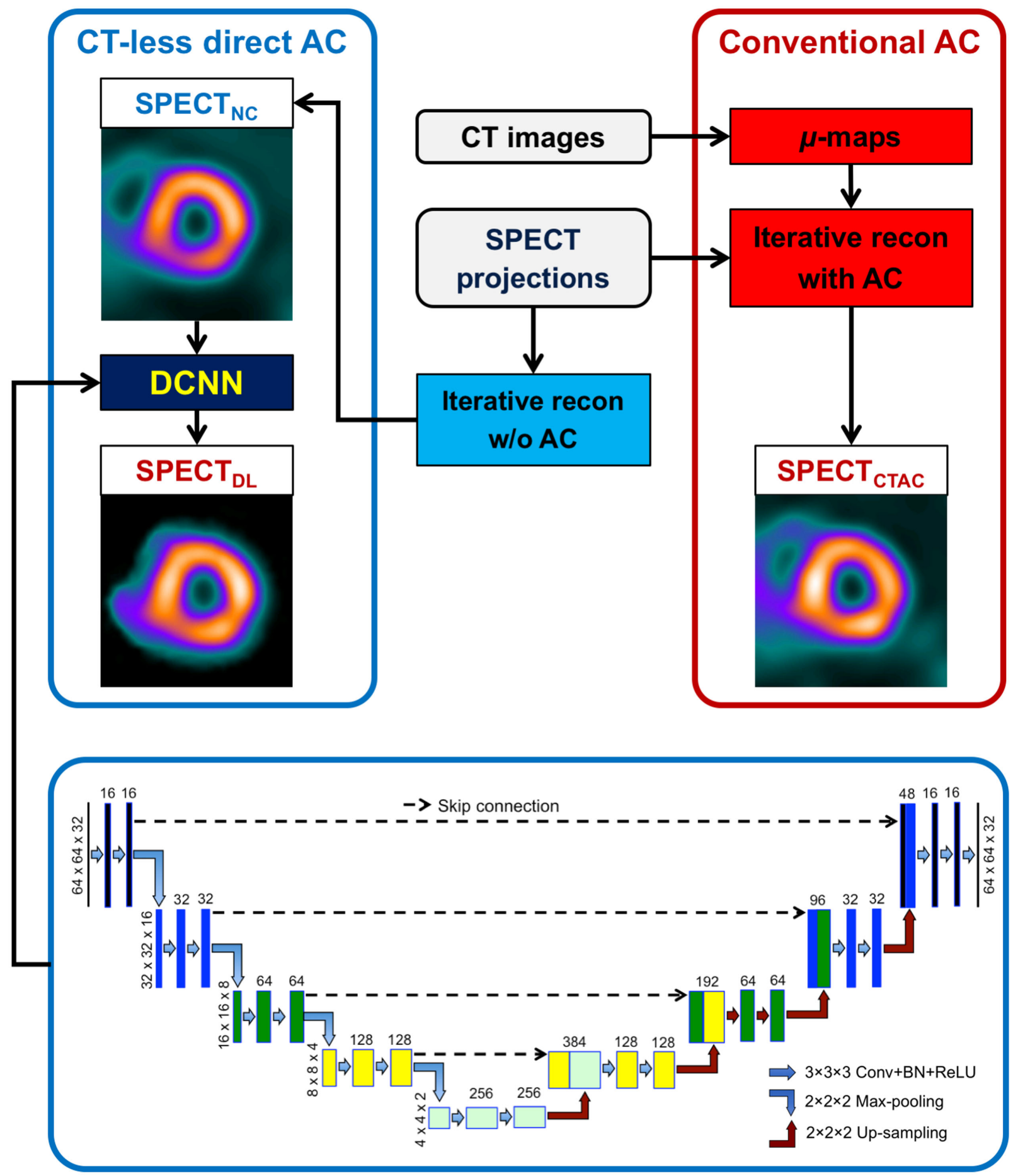

Figure 1. Schematic of a proposed deep convolutional neural networks (DCNN)-based attenuation correction $(\mathrm{AC})$ performed in image space (left), compared to the conventional AC performed through system matrix during SPECT image reconstruction (right) (NC: noncorrected, Conv: convolution; BN: batch normalization; ReLU: rectified linear unit). 
A

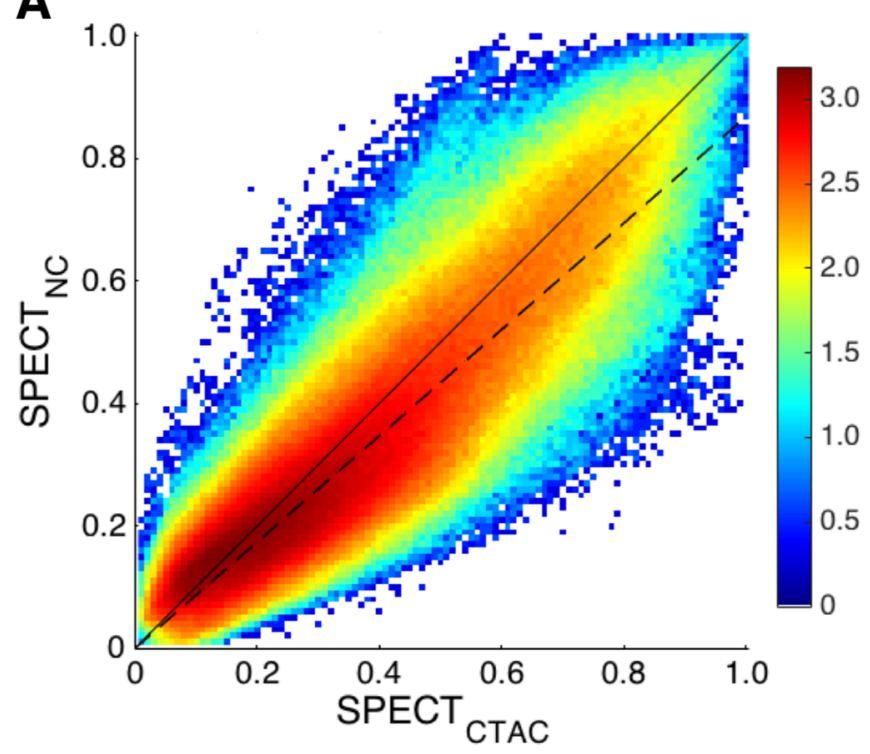

B

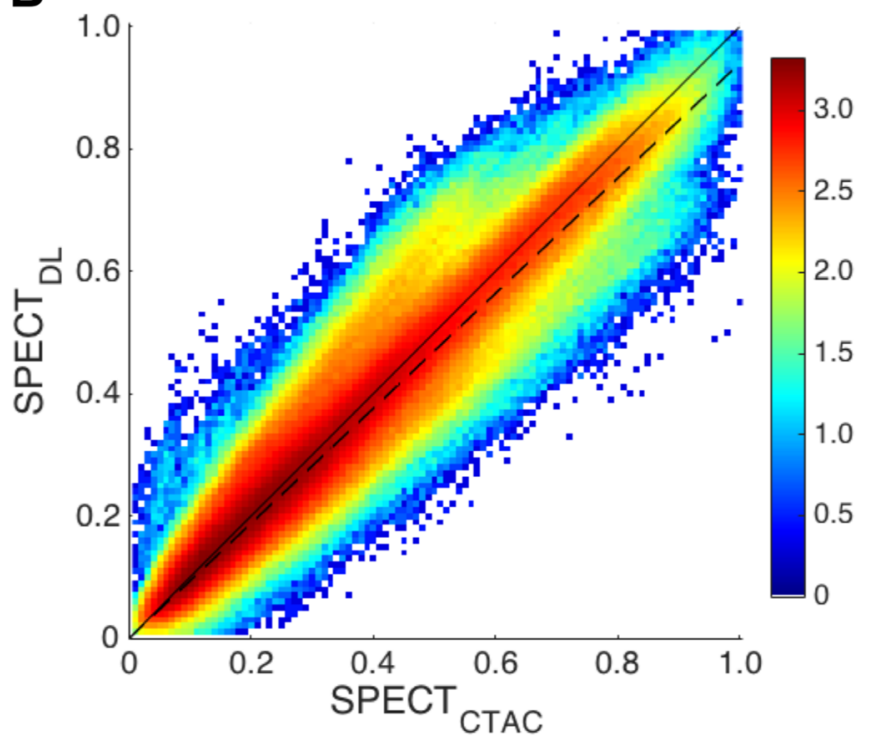

Figure 2. Deep Convolution Neural Network (DCNN) architecture (Conv: convolution; BN: batch normalization; ReLU: rectified linear unit). 
A
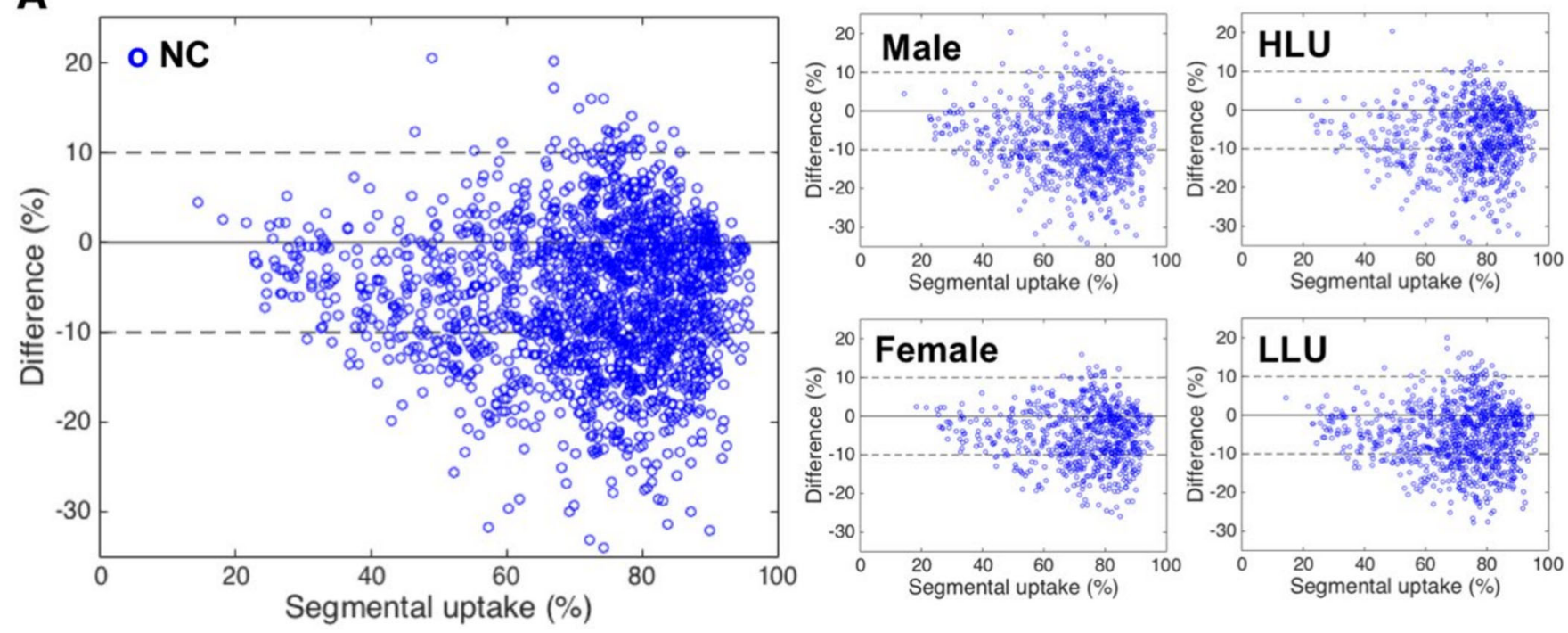

B
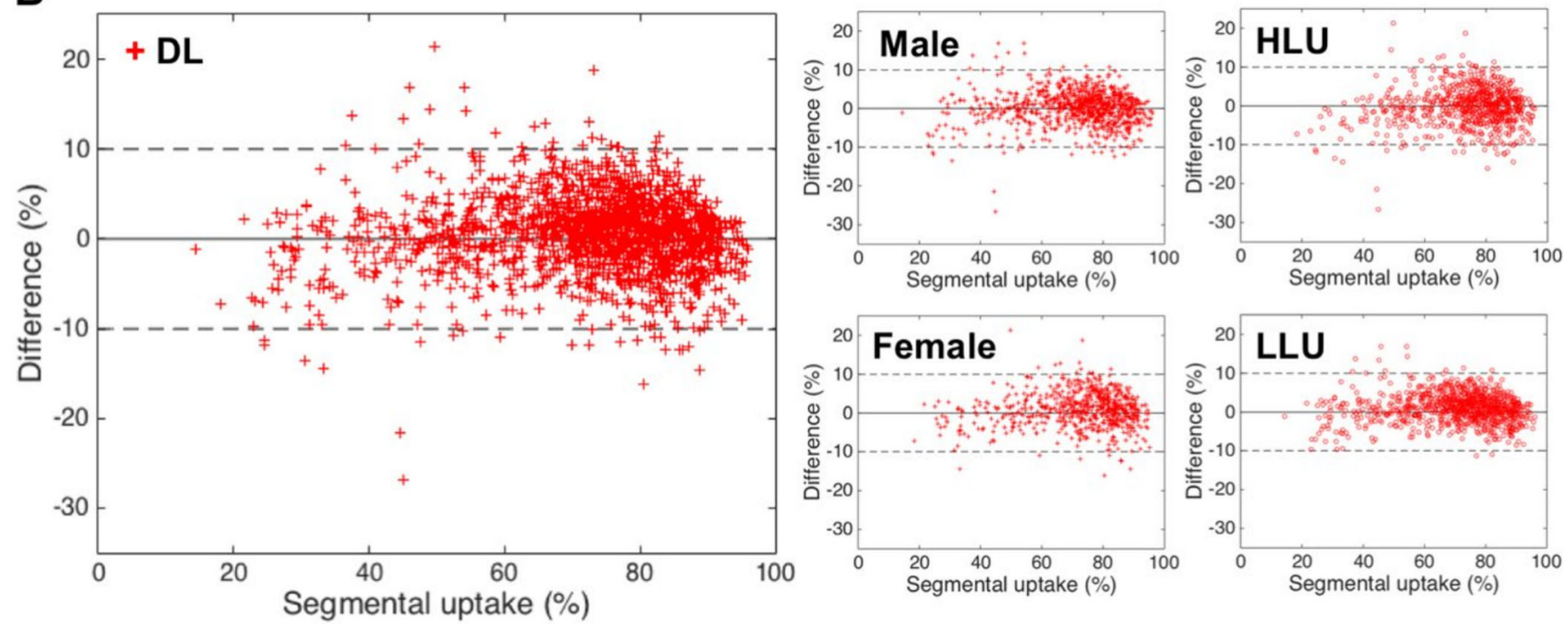

Figure 3. Bland-Altman plots for percentage segmental errors across all subjects, male and female subjects, and subjects with high liver uptake (HLU) and low liver uptake (LLU): SPECTNC (a, blue) and SPECTDL (b, red) (reference: SPECTCTAC). 

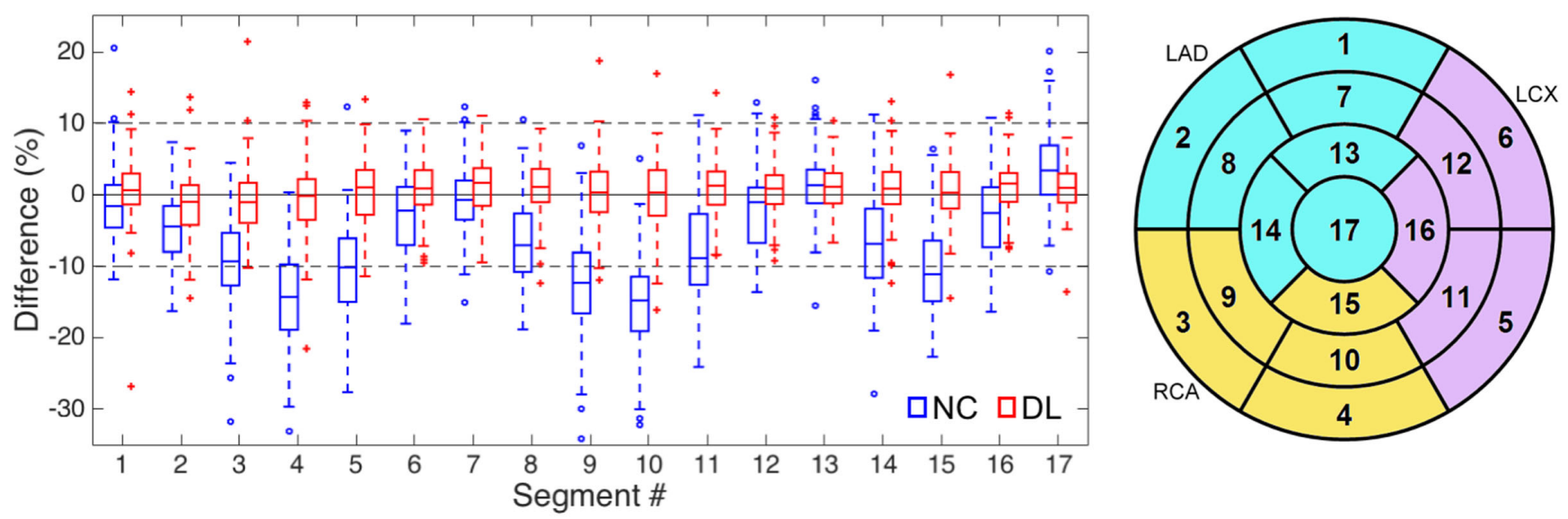

Figure 4. Polar map (a) and box plots for percentage segmental errors across all subjects (b),

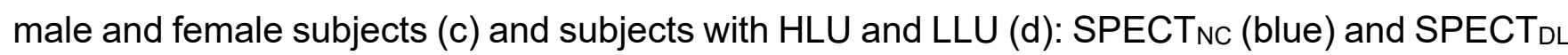
(red) (reference: SPECTсTAC, M: male, F: female, HLU: high liver uptake, LLU: low liver uptake). 
A

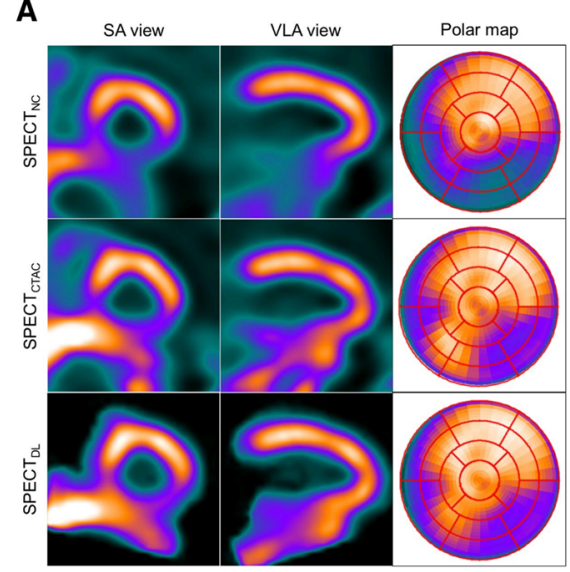

B

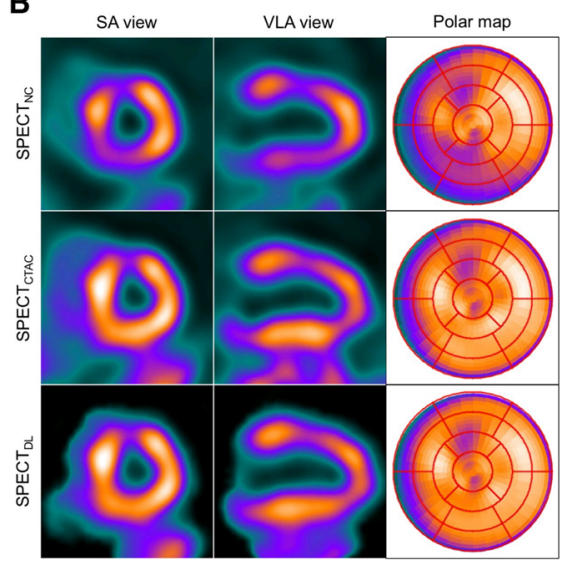

C

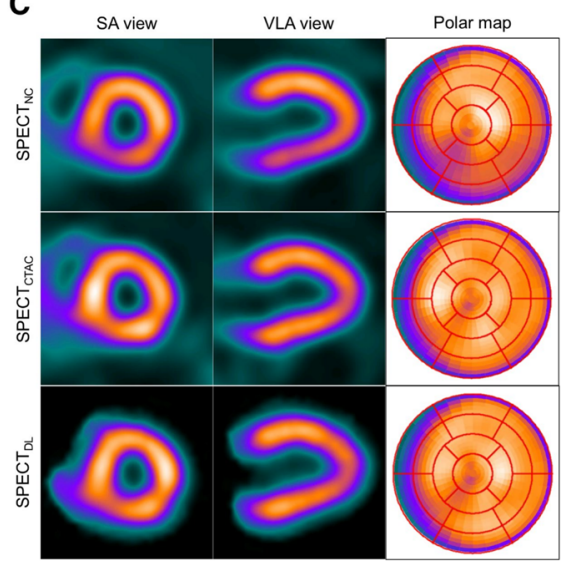

Figure 5. Examples of three subjects ( $\mathrm{A}$, male; $\mathrm{B}$, male; $\mathrm{C}$, male) with the smallest absolute mean segmental error in short axis (SA) and vertical long axis (VLA) views and polar maps:

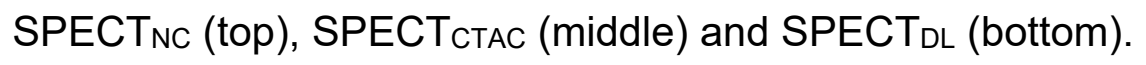


A

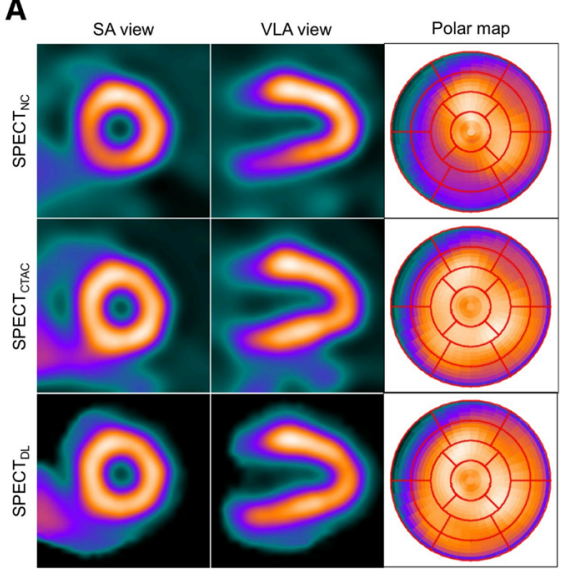

B

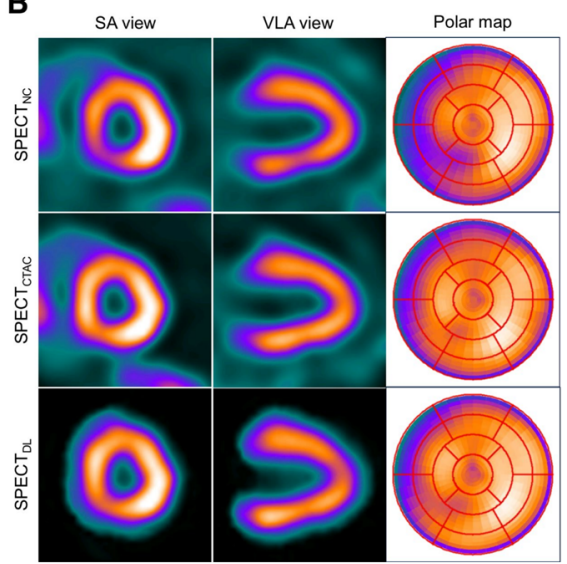

C

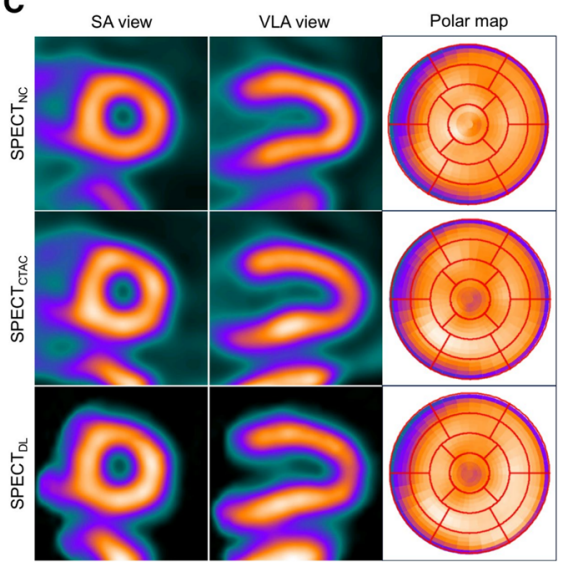

Figure 6. Examples of three subjects with the $25^{\text {th }}(A$, male $), 50^{\text {th }}(B$, male $)$ and $75^{\text {th }}(C$, female $)$ percentiles of absolute mean segmental errors in short axis (SA) and vertical long axis (VLA) views and polar maps: SPECTNC (top), SPECTCTAC (middle) and SPECTDL (bottom). 
A

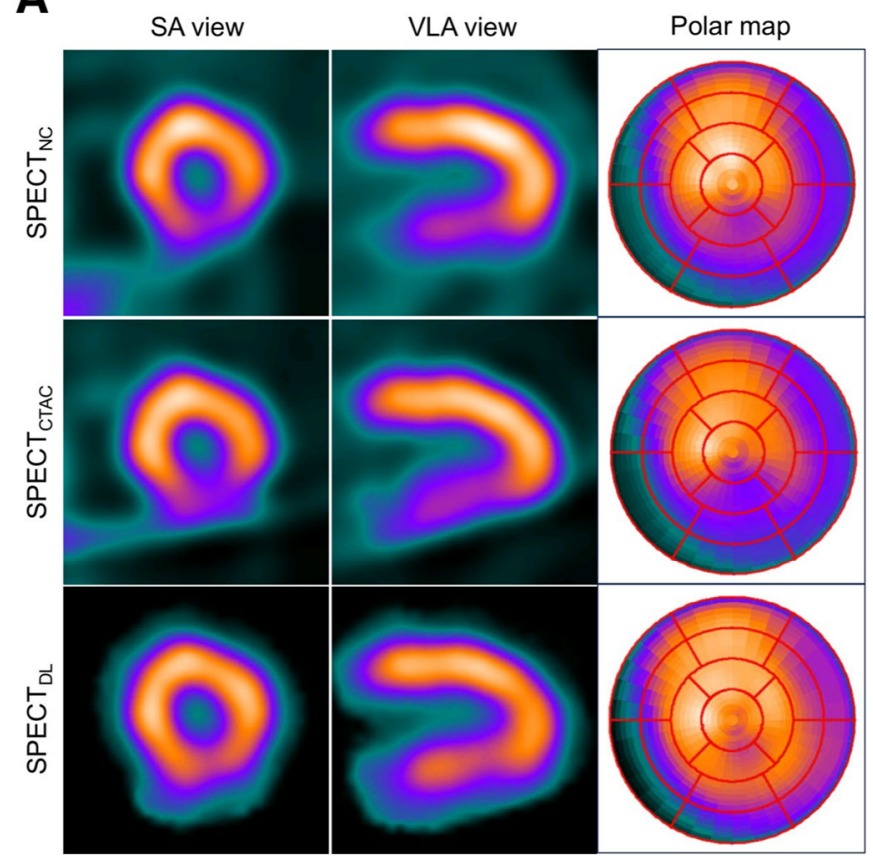

B

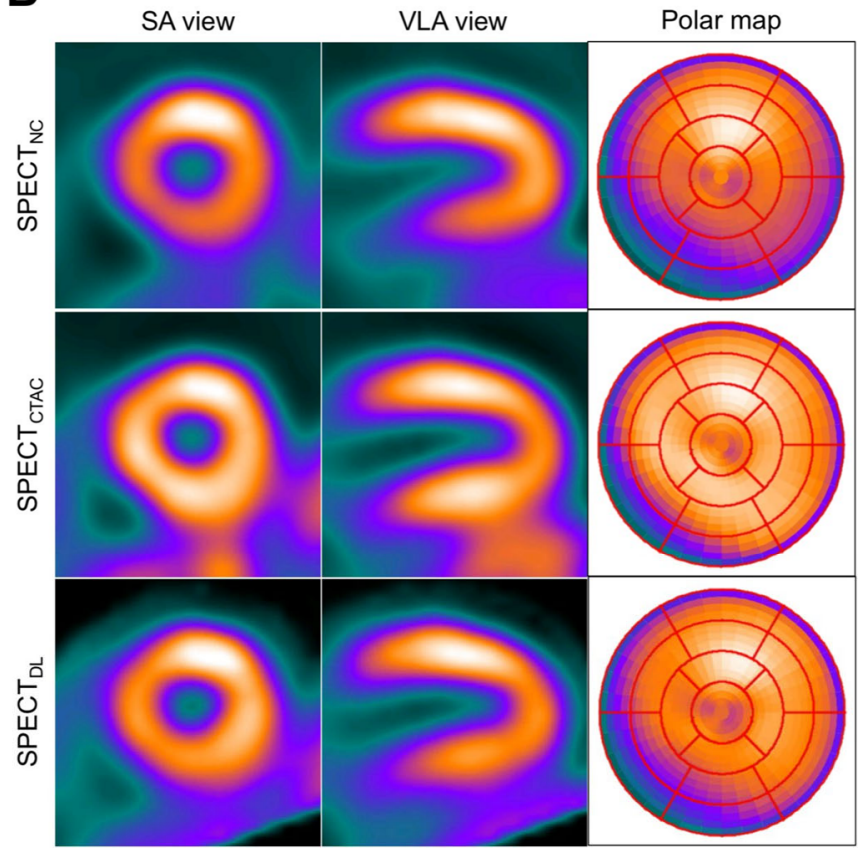

Figure 7. Examples of two subjects with the most overestimated (A, male) and underestimated $(B$, female) mean segmental errors in short axis (SA) and vertical long axis (VLA) views and polar maps: SPECT $\mathrm{NC}$ (top), SPECTCTAC (middle) and SPECTDL (bottom). 
Table 1. Average error, average absolute error and correlation coefficients of all segments across all subjects ( 17 segments $\times 100$ subjects $=1700$ segments in total), 58 males, 42 females, and 44 subjects with high liver uptake (HLU) and 56 subjects with low liver uptake (LLU), respectively. The reference is SPECT ${ }_{\text {CTAC. }} P<0.05$ is considered statistically significant.

\begin{tabular}{|c|c|c|c|c|c|}
\hline \multirow{2}{*}{ Cohort (\#) } & SPECT & $\begin{array}{c}\text { Error (\%) } \\
\text { mean } \pm \text { SD }\end{array}$ & $\begin{array}{c}\mid \text { Error| (\%) } \\
\text { mean } \pm \text { SD }\end{array}$ & Corr. Coef. (\%) & $\boldsymbol{P}$ value \\
\hline \multirow{2}{*}{ All (100) } & NC & $-6.11 \pm 8.06$ & $7.96 \pm 6.23$ & 88.05 & $<0.001$ \\
\cline { 2 - 6 } & DL & $0.49 \pm 4.35$ & $3.31 \pm 2.87$ & 96.17 & $<0.001$ \\
\hline \multirow{2}{*}{ Male (58) } & NC & $-6.96 \pm 8.62$ & $8.80 \pm 6.72$ & 86.66 & $<0.001$ \\
\cline { 2 - 6 } & DL & $0.22 \pm 4.28$ & $3.19 \pm 2.85$ & 96.22 & 0.10 \\
\hline \multirow{2}{*}{ Female (42) } & NC & $-4.95 \pm 7.05$ & $6.80 \pm 5.29$ & 90.36 & $<0.001$ \\
\cline { 2 - 6 } & DL & $0.85 \pm 4.44$ & $3.46 \pm 2.90$ & 96.12 & $<0.001$ \\
\hline \multirow{2}{*}{ HLU (44) } & NC & $-7.30 \pm 8.28$ & $8.71 \pm 6.79$ & 86.72 & $<0.001$ \\
\cline { 2 - 6 } & DL & $-0.21 \pm 4.99$ & $3.75 \pm 3.30$ & 94.75 & 0.25 \\
\hline \multirow{2}{*}{ LLU (56) } & NC & $-5.18 \pm 7.75$ & $7.38 \pm 5.70$ & 89.36 & $<0.001$ \\
\cline { 2 - 6 } & DL & $1.03 \pm 3.69$ & $2.96 \pm 2.43$ & 97.33 & $<0.001$ \\
\hline
\end{tabular}

$\#=$ number of subjects 
Table 2. Selected subjects for Figures 5-7 and their average errors across 17 segments for SPECT DL

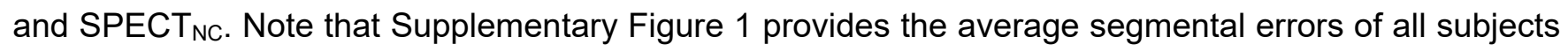
through box plots (HLU: high liver uptake, LLU: low liver uptake).

\begin{tabular}{|c|c|r|r|c|}
\hline Subject \# & Figure \# & DL error (\%) & NC error (\%) & Criteria for DL selection \\
\hline 66, Male, HLU & Figure 5a & $0.10 \pm 4.08$ & $-12.61 \pm 10.04$ & Smallest |error| \\
\hline $\mathbf{5 4 , ~ M a l e , ~ H L U ~}$ & Figure 5b & $0.10 \pm 2.26$ & $-8.73 \pm 8.44$ & Smallest |error| \\
\hline 93, Male, LLU & Figure 5c & $-0.10 \pm 2.99$ & $-6.32 \pm 6.69$ & Smallest |error| \\
\hline 9, Male, LLU & Figure 6a & $1.10 \pm 2.46$ & $-9.04 \pm 7.49$ & $25^{\text {th }}$ percentile |error| \\
\hline $\mathbf{8 9 , ~ M a l e , ~ H L L ~}$ & Figure 6b & $1.99 \pm 3.05$ & $-4.59 \pm 6.85$ & $50^{\text {th }}$ percentile |error| \\
\hline $\mathbf{7 7 , ~ F e m a l e , ~ H L L ~}$ & Figure 6c & $3.15 \pm 4.07$ & $1.17 \pm 5.25$ & $75^{\text {th }}$ percentile |error| \\
\hline 7, Male, LLU & Figure 7a & $9.20 \pm 4.55$ & $2.65 \pm 2.63$ & The most overestimated error \\
\hline $\mathbf{5 7 , ~ F e m a l e , ~ H L U ~}$ & Figure 7b & $-7.21 \pm 4.85$ & $-12.25 \pm 7.25$ & The most underestimated error \\
\hline
\end{tabular}




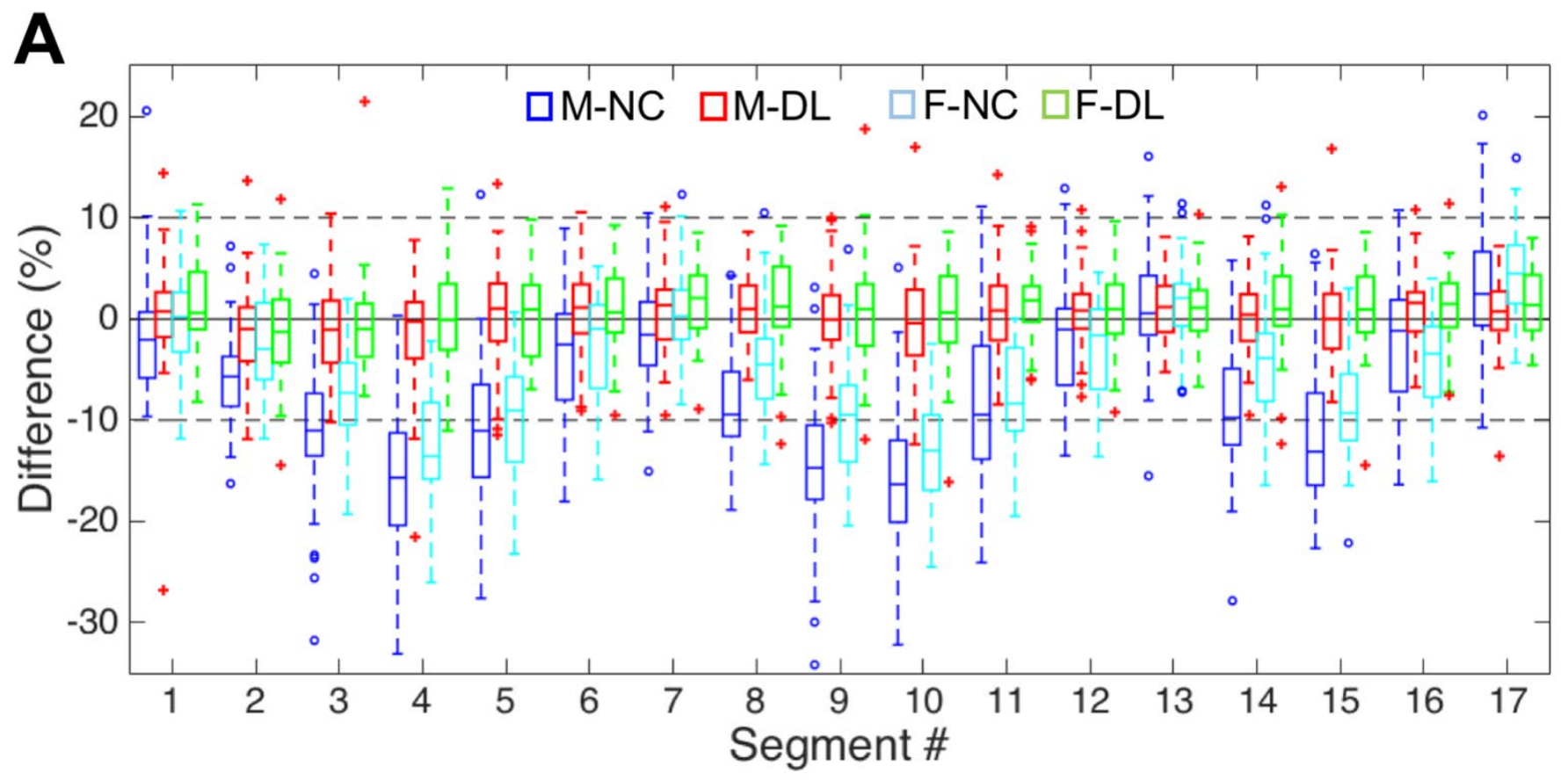

B

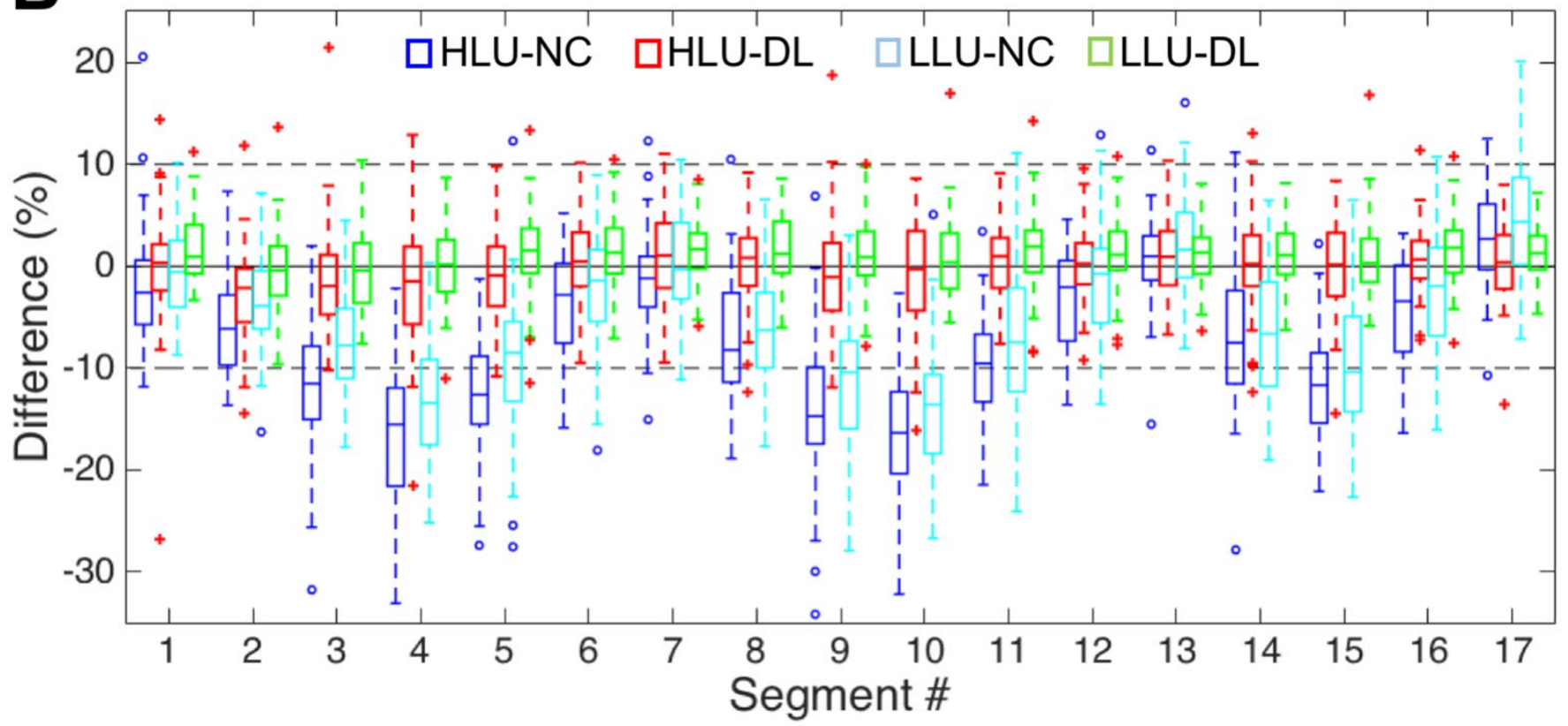

Supplementary Figure 1. Box plots for percentage segmental errors across (A) male and female subjects and (B) subjects with HLU and LLU (reference: SPECTCTAC, NC: non-corrected, DL: deep learning, M: male, F: female, HLU: high liver uptake, LLU: low liver uptake). 
A

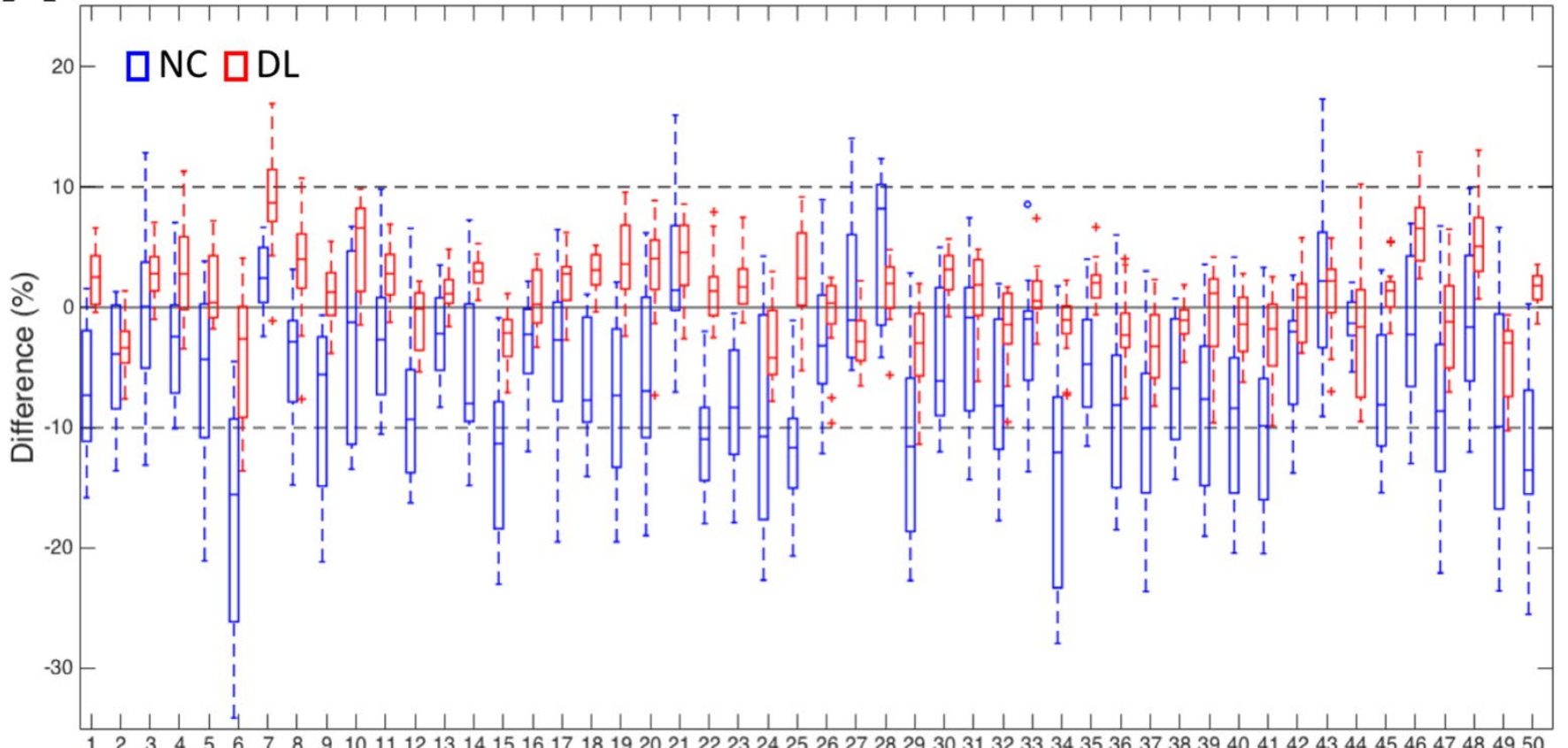

$M F F F M M M M M F M M F M M M F F F F F M M M M M M M M F F M F M F F F F F M F M F M F F F M$ L L L L L H L L L L L L L L L L L H L L L L L L L L H L L L L H L L H H H H H H H

B

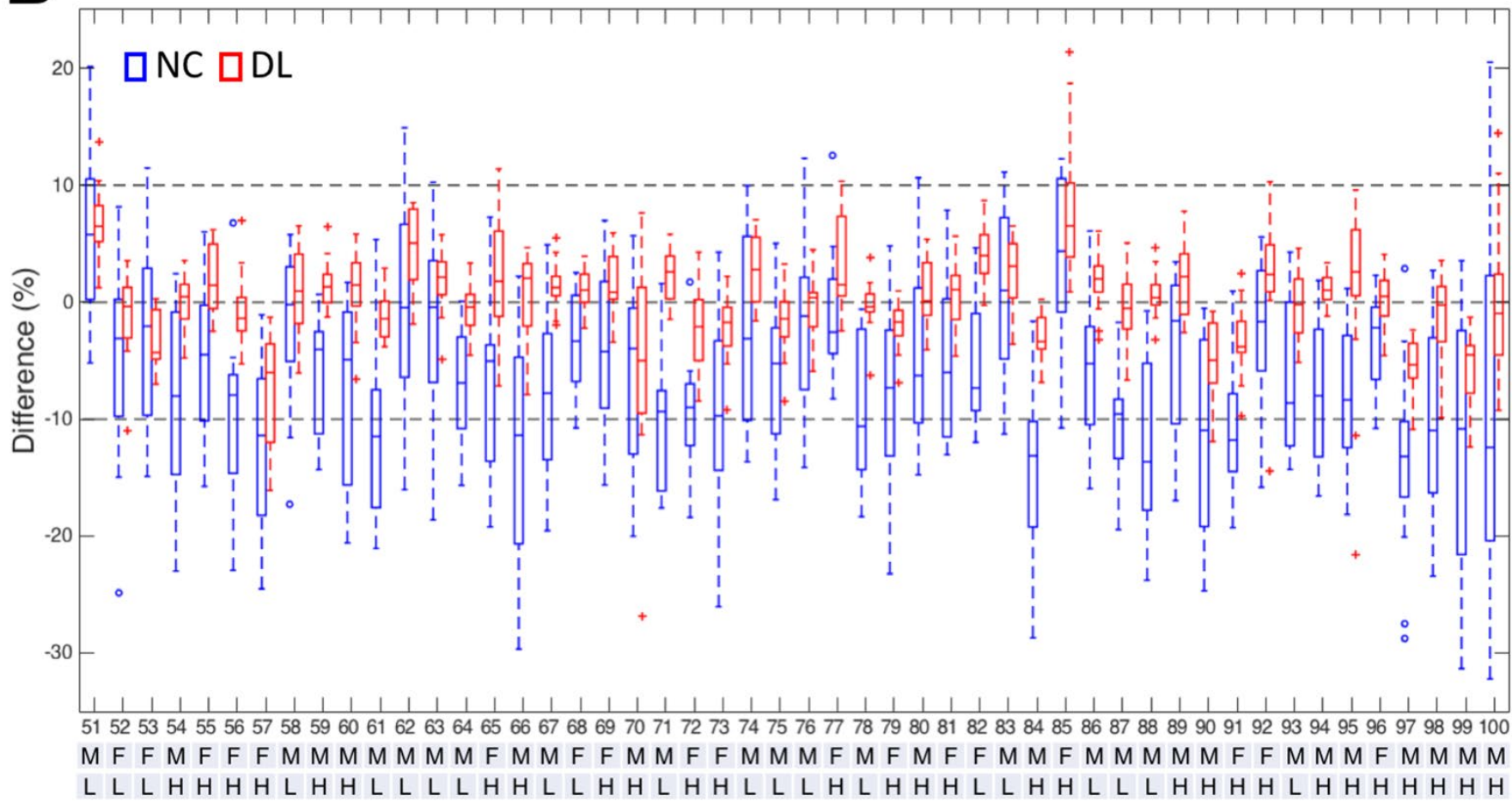

Supplementary Figure 2. Box plots of (A) 1-50 and (B) 51-100 subjects for percentage segmental errors for SPECT $T_{N C}$ (blue) and SPECTDL (red) (reference: SPECTCTAC, M: male, F: female, H: high liver uptake, L: low liver uptake). 
A

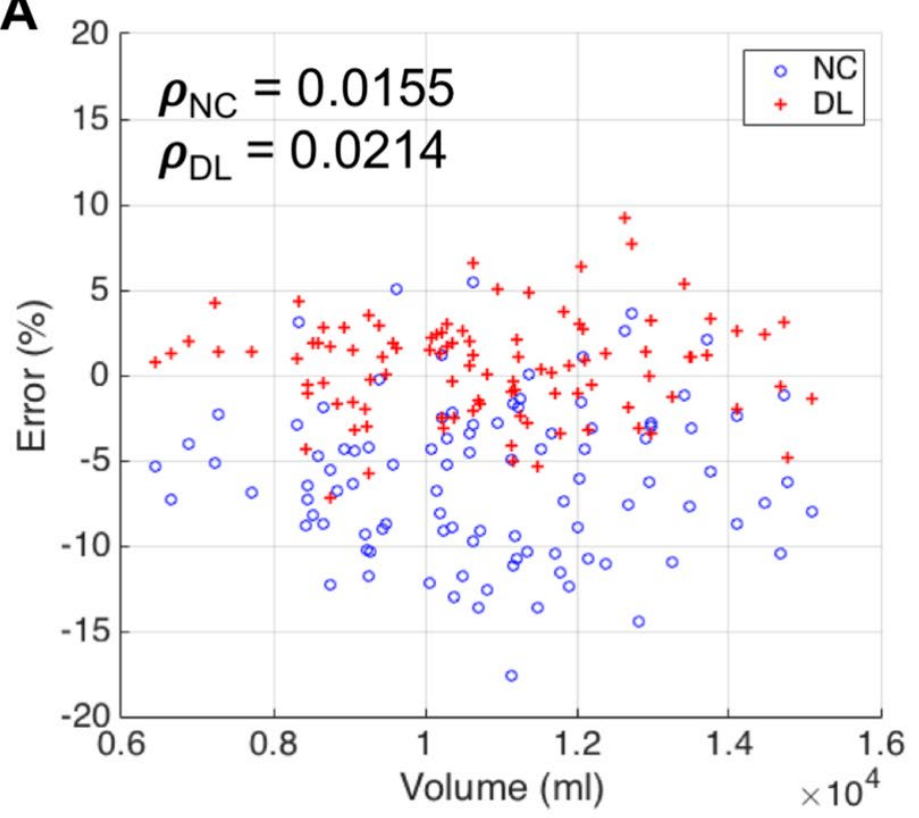

B

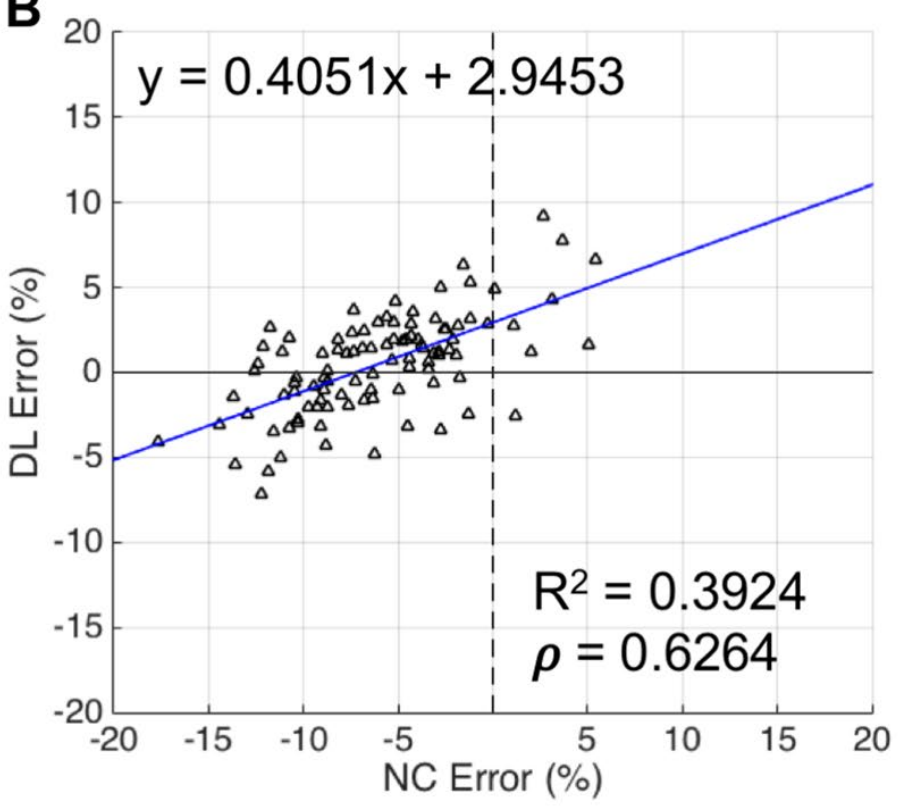

Supplementary Figure 3. (A) Scatter plot of the chest volumes (x-axis) and average segmental errors (y-axis) of SPECT $N C$ (blue) and SPECTDL (red) across 100 subjects and (B) affine regression relation between the errors of SPECTNC (x-axis) and those of SPECTDL (y-axis) across 100 subjects ( $\rho=$ correlation coefficient). 